cavity contained thin fluid with flakes of lymph. A strip of gauze was found leading up among the intestines and another over the pyloric end of the stomach, between it and the liver, from the lower and upper wounds respectively. The cardiac end of the stomach was adherent to the diaphragm and spleen. Between the diaphragm and spleen an abscess cavity was found involving the upper half of that organ. The small intestines were covered with recent lymph. The liver was large and pale; it weighed 4 pounds $1 \frac{1}{2}$ ounces ; on section it was paler and softer than normal and showed signs of decomposition. The gall-bladder was full of thick yellow bile and was normal. The left kidney weighed four ounces ; its cortex was somewhat diminished in extent; it was pale, the capsule stripped off, leaving a rough surface, and showed some cysts. The right kidney weighed three and three-quarter ounces; it was similar to the left. The spleen was slightly enlarged (about half as large again as normal), its capsule was thickened, and its upper half contained an abscess cavity with black sloughing walls. This abscess was separated from the spleen substance below by a thick fibrous wall. The lower half of the spleen contained several small infarcts. Microscopical examination showed numerous streptococci in the pus. (These were afterwards obtained in pure culture.) The pancreas was normal and weighed four ounces. The stomach was slightly congested at the cardiac end; it was not ulcerated. The small intestines were healthy except for some congestion at the lower part of the ileum. The large intestines were healthy. 'The vermiform appendix was two and a half inches long and normal. The bladder was normal. The uterus was enlarged; it was four inches long and its cavity contained some recent blood clots. There was some roughening of the anterior wall at the site of the placenta; it was healthy. The ovaries were normal. The cause of death was pyæmic abscess of the spleen and peritonitis after child-birth.

Remarks. - The case appeared to be one worthy of recording, as I believe a single abscess of the spleen with no suppuration in other organs is a rare affection. The most complete paper having a bearing on the subject that I am able to lay my hands on at the present time is one on Splenic Infections by Stavely in Annals of Surgery for 1903. He refers to a considerable number of recorded cases, but none of them appear to have followed, or been connected with, puerperal conditions.

It seems almost certain that the original infection must have started from the uterus and the extremely offensive state of the liquor amnii, taken with the fact that the lochia were also offensive from the first day, would point to some form of ante-partum infection from which the genital organs appeared to have recovered, but not before the infection had been carried to the spleen in the form of a septic embolus.

In this case there were absolutely no local indicationssuch as swelling, fluctuation, or redness-to be detected, the only symptom being diffuse tenderness over the lower ribs and hypochondriac region on the left side, though from the post-mortem appearances the abscess must have existed for some time. As to the influence of the eclamptic condition on the case it must undoubtedly greatly have lessened the chances of recovery. The state of the kidneys found at the post-mortem examination would seem to indicate that there was a previous nephritis of a chronic nature and this probably accounted for the fact that albumin was present in the urine up to the last.

My best thanks are due to Lieutenant-Colonel F. S. Peck, I.M.S., for his kind permission to publish the case ; to Captain Leonard Rogers, I.M.S., acting professor of pathology at the Medical College, for the full and complete notes of the post-mortem examination; and to Captain E. Owen Thurston, I.M.S., resident surgeon, Medical College Hospital, for the kind assistance he gave me at the operation.

Wimbledon and District Medical Society.The following are the names of those elected as officers of the above society for the session 1905-06:-President: Dr. Martin Randall. Vice-Presidents : Mr. Gr. Walker and Dr. H. Love. Conamittee: Mr. G. W. Brabyn, Dr. S. R. Collier, Dr. D. Findlay, Dr. R. Richmond, and Mr. P. B. Spurgin. Honorary treasurer: Dr. J. Edwin Bates. Honorary secretary: Mr. F. Deas. The meetings of the society will be held at Wrirple Hall, Worple-road, Wimbledon, and commence at 9 P.M. Members are invited to bring their medical friends.

\section{SOME REMARKS ON THE GERM CENTRES OF LYMPHATIC GLANDS AND SECONDARY CARCINOMATOUS DEPOSITS.}

By J. BURTON CLELAND, M.D., Ch.M.Syd., CANCER RESLARCH SCHOLAR, LONDON HOSPITAL. (From the Pathological Institute, London Hospital.)

A CLOSE resemblance often exists between the "germ centres" of lymphatic glands and secondary carcinomatous deposits. There is no doubt, further, that the former have at times and in competent hands been mistaken for the latter and that cases in which sections from the primary site have left the question of malignancy unsettled have been finally classified as such on the finding of these apparently secondary deposits in the adjacent lymphatic glands. This is little to be wondered at for several reasons. First, the text-books have in general but little to say on the various appearances presented by these centres and none, so far as I am aware, on the question of distinguishing them from a secondary growth. The general observer is ignorant of their presence and the specialist only too frequently has imperfectly fixed material on which to decide. Secondly, even with perfect and immediate fixation and careful staining, it is sometimes exceedingly hard to say which is canerous growth and which a germ centre, though undoubted examples of each of these may be at hand for comparison.

It may be well, perhaps, first briefly to indicate the different appearances that the germ centres may present. The cells of which they are composed are larger than the surrounding lymphocytes and have more protoplasm and a larger and vesicular nucleus; the whole area stains, too, to a much lighter degree than the parts around. Mitotic figures are nearly always numerous and at the edge of the area the cells rapidly, but by distinct gradations, become converted into lymphocytes, so that no hard and fast line of demarca. tion limits the area. The areas are usually small and indistinctly circumscribed and in such conditions are not likely to be mistaken for a cancerous growth. In certain circumstances, however, associated with chronic irritation, they assume a most striking appearance. This is associated with increase of size and great increase of mitotic activity in the areas and suggests the idea that it is a form of com. pensatory hypertrophy. For example, the most marked instance of it which $I$ have ever seen was in a small gland from the neck of a child (Fig. 5) in which one-half of the gland was occupied by a fibrotic tuberculous area and the healthy half exhibited a great number of these hypertrophied organs, as if compensating for the partial destruction of the gland. Again, close to a very early secondary deposit from a carcinoma of the breast occurred a very marked instance of the enlarged germ centres. (See Figs. 1 to 4.) Further, in a mesenteric gland, riddled with small secondary deposits from a carcinoma of the stomach, each of the islands of lymphatic tissue still left had its centre composed of a proliferating germ centre and the periphery of lymphocytes formed from this, the appearance suggesting, which was doubtless true, that each germ centre was doing its utmost to make good the loss of lymphatic tissue elsewhere in the gland and was partially successful in its efforts.

These hypertrophied germ centres form very sharply defined spherical or oval areas, taking a much lighter stain than the parts around and about as deep as that of an epithelial deposit from the breast. The cells composing them are of nearly uniform size and a little smaller and much more regular than those of cancer; they are closely packed together with very little stroma detectable between them, though an occasional capillary vessel may be noticed; the nucleus resembles closely that of an epithelial cell; and mitoses are often mole numerous or as numerous as in the cancerous growth. All these characters show how hard it may be to distinguish the one from the other. The most important difference between the two is, however, found in the boundary zone of each. The somewhat larger and more irregular cells of the carcinoma are separated sharply and distinctly and often by a thin zone of fibrous stroma from the surrounding lymphocytes and each individual cell at the periphery can be 
FIG. 1.

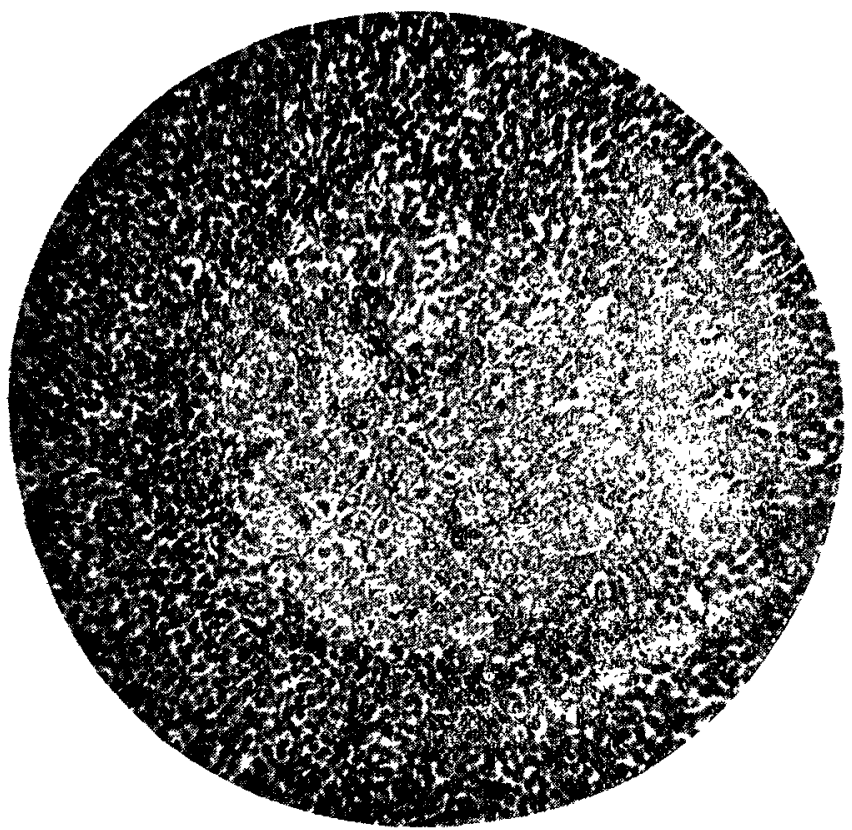

FIG. 2.

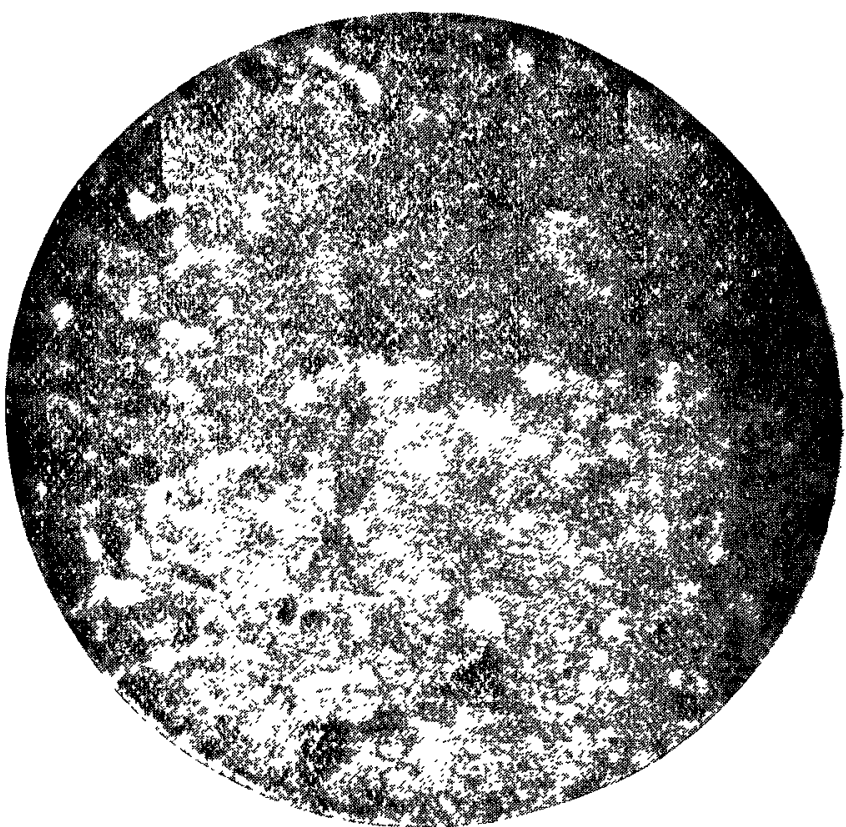

FIG 5

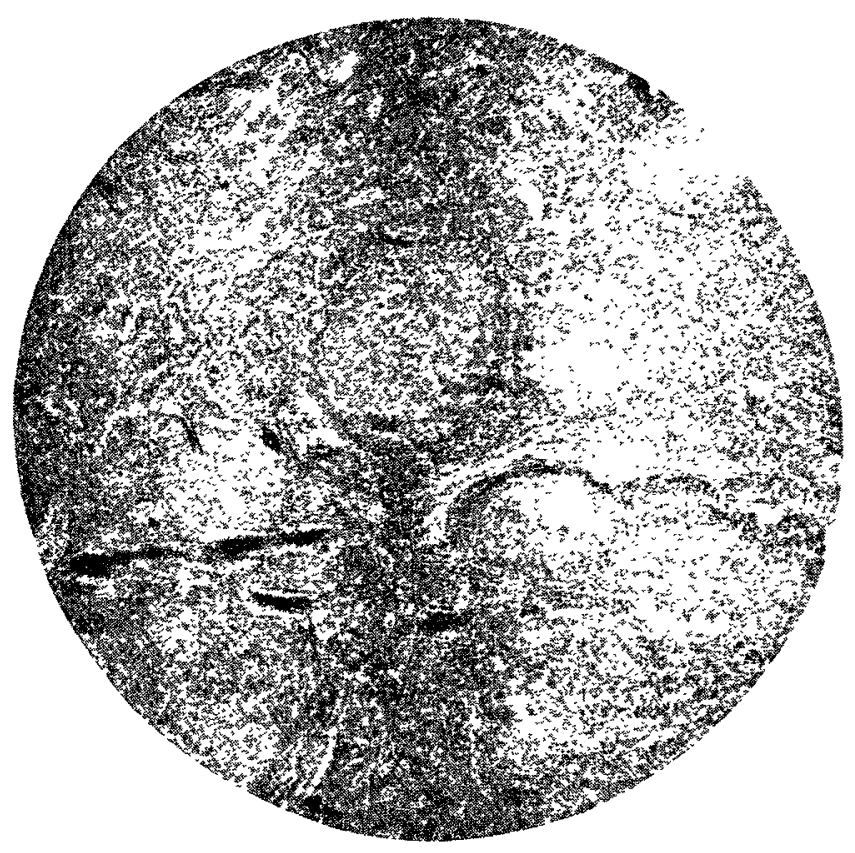

FIG. 3.

FIG. 4.
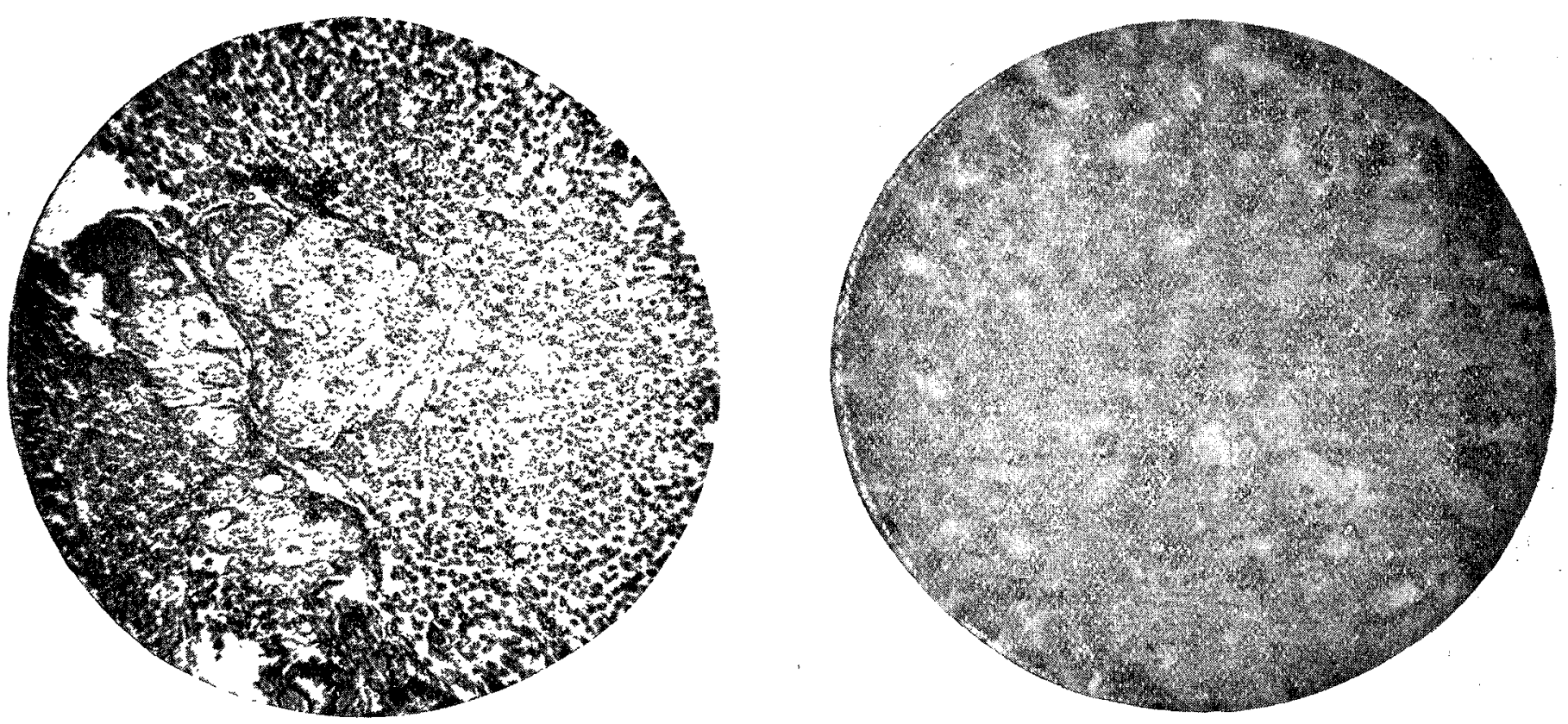

Figs. I to 4. Sections from enlarger lymphatic gland in carcinoma of the breast. Figs. 1 and 2. Germ centre. Fig. $2 \times 667$ diameters. Fig. 5 . Enlarged germ centres. I 4 mphatic oland of a child. $\times$ about 60 dianseters.

(From micro-photographs by Mr. E. Wilson, photographic depariment, Lcné on IIospital.) 
relegated at once and satisfactorily into one or other class of these two. Not so is it, however, in the outer zones of the germ centres. Here both germ centre cells and lymphocy tes tend to be arranged in concentric rings formed of a delicate fibrous tissue and all gradations are seen between undoubted germ-centre cells, on the one hand, and undoubted lymphocytes on the other. Under a low power of magnification the germ centre often seems sharply delimited from the surrounding parts and the cancerous deposit is lens distinctly outlined; with higher magnifying powers this appearance is reversed and the germ centre is found, on careful observation. to grade into the lymphatic tissue around while the carcinomatous growth is sharply defined.

If during an examination of the lymphatic glands in a doubtful case of carcinoma the possible presence of these hypertrophied centres be borne in mind, it is not likely that any mistake will be made; but should an area of cells be encountered which gives rise to any doubt a careful consideration of the various points of difference enumerated above will enable the observer to satisfy himself as to its nature and may relieve also the mind of the surgeon and of his patient of the distressing dread of a recurrence of a disease which is not there.

\section{ANEURYSMAL DILATATION OF THE RIGHT SUBCLAVIAN ARTERY, THE INNOMINATE AND THE FIRST PART OF THE COMMON CAROTID ;}

DISTAL LIGATURE OF THE FIRST PART OF THE AXILLARY ARTERY AND THE MIDDLE OF THE COMMON CAROTID WITH RESULTING OBLITERATION OF THE SUBCLAVIAN.

BY H. GILBERT BARLING, M.B., B.S. LOND., F.R.C.S. ENG.,

SURGEON TO THE GENERAL HOSPITAL, BIRMINGHAM.

A FINELy built man, aged 45 years, was admitted to the General Hospital on Sept. 27th, 1904, complaining of pain in the right upper extremity and a pulsating swelling above the right clavicle. The pain in the right arm began about six months before admission; it was of an aching character and very continnous, the most troublesome part being the right shoulder blade. The patient found that he was unable to sleep on his right side. Pain was the first symptom noticed and three months later a swelling appeared above the clavicle; this gradually got larger and for two months the patient sought relief by carrying his arm in a sling.

On admission a swelling of about the size of an egg, with expansile pulsation and a soft bruit over it, was found above the right clavicle. The swelling extended from the outer margin of the sterno-mastoid along the subclavian artery as far as the latter could be examined; the other accessible vessels appeared to be quite healthy but pulsation was slightly delayed in the right radial artery. Cardiac dulness appeared to be normal but there was a doubtful murmur in connexion with the first sound over the aortic area and the second sound was ringing in character. There did not appear to have been any special injury or severe strain as the cause of the aneurysm; the patient had never suffered from specific disease, but he had been the subject of several attacks of gout and there were numerous tophi in both ears. The condition was regarded as one of aneurysmal dilatation of the second and third parts of the subclavian artery rather than as a distinct sacculated aneurysm. For the treatment alternative courses presented themselves : (1) ligature of the first part of the subclavian; (2) ligature of the innominate artery; whilst a third possibility was not at that time contemplated-namely, distal ligature of the first part of the axillary artery. On the whole it appeared that, grave as is the ligaturing of either of the arteries above mentioned, experience was in favour of tying the innominate and it was determined to endeavour to secure this vessel. An incision was accordingly made along the internal border of the sterno-mastoid, beginning about its middle and extending downwards over the sternoclavicular joint; from the lower end of this a second incision nearly three inches was made along the clavicle. The fap of skin was turned back, both heads of the muscle were separated from the bones, the sterno-hyoid and sternothyroid muscles were divided and drawn inwards, and some veins of considerable size were ligatured. The hope of being able to expose the innominate without removal of any part of the clavicle was soon shown to be vain and accordingly the inner third of the clavicle was removed. The internal jugular vein being drawn outwards careful dissection expored the lower part of the carotid artery where it ended in the innominate and it was at once evident that the whole of the innominate and also three-quarters of an inch of the carotid artery were widely dilated and were undesirable vessels for the application of a ligature. Attention was then directed to the possibility of ligaturing the first part of the subclavian artery and the vertebral so as to diminish as far as possible the disturbance of the blood current close to the ligature if the first part of the subclavian was chosen. Here, again, disappointment was met with, for the first part of the subclavian was equally dilated as the other vessels exposed and the vertebral artery also ; it was evident therefore that one had to deal with marked dilatation of the innominate, the first portion of the common carotid, and of the whole of the subclavian artery and possibly of all the main branehes of the last mentioned. With the concurrence of my colleague Sir T. F. Chavasse, who was good enough to help me at the operation, determined to ligature the common carotid artery above the limit of dilatation and to tie also the first part of the axillary if that were found to be healthy. The last-mentioned vessel was exposed at its highest part and was found healthy; this and the middle of the common carotid were therefore ligatured with kangaroo tendon somewhat coarser than No. 4 catgut. Each ligature, a single one, was tied with the intention of using such force as was necessary to close each vessel but without dividing the internal coat of either. The wound was drained by a narrow drainagetube which was placed just under the flap of skin and not in close proximity to either ligature; it seemed safer to drain as the wound was rather oozy, firm compression being not easy to obtain. The wound healed throughout by first intention but the patient was kept at rest for nearly five weeks and when he was discharged from the hospital his right arm was bandaged to his side, though a little later on he was allowed to use the hand for writing so that he might carry on his work as an agent. The arm was kept to the side until May 18th, when he was allowed to dispense with the bandage and to use the limb more freely.

At the present time there is no pulsation to be felt in any part of the subclavian artery, a quite small current of blood finds its way into the lowest portion of the right common carotid, and it is doubtful whether the innominate can be felt ; certainly nothing like a dilated artery is within reach.

The surgical treatment of diseases of the large arteries assumes in the present day a less important position than that assigned to it in the past. There are probably two main reasons for this, the first being the more frequent occurrence of other great lesions of the body to the successful treatment of which surgeons have devoted so much time and thought during the past few years These new fields of surgery from which our predecessors were excluded have proved so interesting and so productive of great results that they have drawn attention away from the more classical subjects such as the ligature of great arterial trunks. A second explanation of this lessened interest may be found in the fact, if fact it be, that aneurysm of the large arteries accessible to surgical interference is less common now than formerly. This is distinctly the impression left on my mind from what $I$ have seen in this hospital during the last 25 years and $I$ think it is an impression shared by many surgeons.

The changed conditions under which operative work is now undertaken, as compared with the past, have nowhere had a greater influence than in arterial ligature in continuity. Success depends in very great measure on two factors, the use of a trustworthy ligature, which is absorbed when its work is done, and healing of the wound by first intention. The full influence of the work of Pasteur and Lister is hardly yet known to us so far as it applies to the ligature of main arteries in the treatment of aneurysm. Owing to the infrequency of such operations experience accumulates slowly 